\title{
Whole-Plant Corn Silage Improves Rumen Fermentation and Growth Performance of Beef Cattle by Altering Rumen Microbiota
}

Yalei Cui

Henan Agricultural University

Hua Liu

Henan Agricultural University

Zimin Gao

Henan Agricultural University

Junying $X u$

Henan Agricultural University

Boshuai Liu

Henan Agricultural University

Ming Guo

Henan Agricultural University

Xu Yang

Henan Agricultural University

Jiakuan Niu

Henan Agricultural University

Xiaoyan Zhu

Henan Agricultural University

Sen Ma

Henan Agricultural University

Defeng Li

Henan Agricultural University

\section{Yu Sun}

Henan Agricultural University

Yinghua Shi ( $\square$ annysyh@henau.edu.cn )

Henan Agricultural University https://orcid.org/0000-0003-0516-4234

\section{Research}

Keywords: roughage, whole-plant corn silage, beef cattle, rumen fermentation, microbiota 
Posted Date: October 22nd, 2021

DOl: https://doi.org/10.21203/rs.3.rs-944720/v1

License: (c) (i) This work is licensed under a Creative Commons Attribution 4.0 International License. Read Full License 


\section{Abstract}

\section{Background}

In recent years, whole-plant corn silage had been widely used in China. Roughage was an important source of nutrition for ruminants and had an important effect on rumen microbiota, which plays an important role in animal growth performance and feed digestion. To better understand the effects of different silages on rumen microbiota, the effects of whole-plant corn silage or corn straw silage on growth performance, rumen fermentation products, and rumen microbiota of Simmental hybrid cattle were studied.

Results

60 healthy Simmental hybrid cattle were randomly divided into 2 groups with 6 repeats in each group and 5 cattle in each group. They were fed with whole-plant corn silage (WS) diet and corn straw silage (CS) diet respectively. Compared with corn straw silage, whole-plant corn silage significantly increased daily gain and decreased feed-weight ratio of beef cattle. Whole-plant corn silage also decreased the acetic acid in the rumen and the acetate to propionate $(\mathrm{A} / \mathrm{P})$ ratio compared with corn straw silage. At the genus level, the relative abundance of Prevotella_ 1 was significantly increased while the relative abundance of Succinivibrionaceae_UCG-002, Succiniclasticum, norank_f_Bacteroidales_RF16_group, and Ruminococcus_ 1 was decreased in cattle fed whole-plant corn silage compared with those fed corn straw silage. Prevotella_ 1 was positively correlated with acetic acid and A/P ratio, Succinivibrionaceae_UCG002 was positively correlated with propionic acid and butyric acid, and negatively correlated with $\mathrm{pH}$, Succiniclasticum was positively correlated with $\mathrm{pH}$ and A/P ratio, and norank_f_F082 and Rikenellaceae_RC9_gut_group were positively correlated with $\mathrm{pH}$, propionic acid and butyric acid. Feeding whole-plant corn silage improved amino acid metabolism, nucleotide metabolism, and metabolism of beef cattle compared with feeding corn straw silage. Correlation analysis between rumen microbiota and metabolic pathways showed that Succinivibrionaceae_UCG-002 was negatively correlated with Carbohydrate Metabolism, Glycan Biosynthesis, and Metabolism, while Prevotellaceae_UCG-003 was positively correlated with Amino Acid Metabolism, and Carbohydrate Metabolism.

Conclusions

Feeding whole-plant corn silage can improve the production performance rumen fermentation of beef cattle by altering rumen microbiota, amino acid metabolism, and nucleotide metabolism.

\section{Introduction}

Roughage plays an important role in ruminant feeding. For a long time, corn straw has been used as an important source of roughage for ruminants, especially in beef cattle production. Corn straw is rich in carbohydrates, which be used for fermentation of silage, but its heavily lignified cell walls lead to low digestibility, and low nutritional value, making it difficult to meet the needs of ruminant production with 
corn straw alone. Whole-plant corn silage is a high-quality source of roughage. It contains a considerable amount of corn kernels and is usually used to replace the concentrate part of the diet, playing a nutritional role similar to that of concentrated fodder [1]. Production practice shows that whole-plant corn silage possesses rich nutrition, high feeding value, and can well meet the production needs of ruminants $[2,3]$. Zaralis et al. [4] showed that the daily gain of beef cattle fed with whole-plant corn silage alone was higher than that of cattle fed whole-plant corn silage and forage silage. Meanwhile, Hameleers [5]demonstrated that whole-plant corn silage can effectively increase milk yield, improve milk quality, reduce breeding costs, and increase economic benefits.

Improving animal growth performance manipulating rumen microbiota and ruminant metabolism through diet alterations has gained increasing attention during recent years. The rumen is a digestive organ unique to ruminants, and plays an important role in the whole digestive process [6]. Ruminants use microbiota in the rumen to ferment and degrade nutrients such as cellulose and hemicellulose in rouge to form volatile fatty acids (VFAs), such as acetic acid, propionic acid and butyric acid [7]. VFAs are the main source of energy for ruminants, providing $60 \%$ of the energy needed by the body [8]. VFAs produced by rumen fermentation are partly absorbed by the rumen epithelium, partly neutralized by saliva, and partly absorbed as the chyme enters the small intestine. Absorption and utilization of VFAs play an important role in the life activities of ruminants. Kolver et al.[9]showed that increasing the proportion of concentrate in the diet will increase the concentration of VFAs, thus improving production performance.

Rumen microbiota is a relatively stable but continuously dynamic community. The composition and distribution of rumen microbiota are affected by diet, season, host health, environmental temperature, humidity, and other factors [10]. The studies of Jami et al. [11] and Huws et al [12] show that rumen microbiota has an important effect on the growth, health, and immunity of the host. For healthy growing cattle, dietary composition is the most important factor affecting the rumen microbial community, and the rumen microbiota and their metabolites will change when cattle are fed with different diets [13]. The purpose of this study was to investigate the effects of whole-plant corn silage and corn straw silage on growth performance, rumen microbiota, and metabolites of Simmental hybrid cattle.

\section{Results}

\subsection{Effects of different diets on growth performance of beef cattle}

Beef cattle were fed either whole-plant corn silage (WS) or corn straw silage (CS) for 80 days. The effects of different treatments on the growth performance of beef cattle are presented in Fig.1. There were no difference in ADFI between diets $(P>0.05)$. The ADG of cattle under the WS treatment was significantly higher than that of cattle under the CS treatment, and the F: G ratio under the WS treatment was significantly lower than that under the CS treatment $(P<0.05)$.

\subsection{Effects of different diets on the concentration of VFAs in beef cattle rumen}


The effects of different diets on rumen VFAs of beef cattle are presented in Fig.2. Rumen pH values in both treatments were in the normal range, and there was no significant difference in the total epigenetic fatty acids $(P>0.05)$ while the acetic acid content in the CS treatment was significantly higher than that in the WS treatment $(P<0.05)$. The ratio of acetic acid / propionic acid in the WS treatment was significantly lower than that in the CS treatment $(P<0.05)$.

\subsection{Effects of different diets on the diversity and composition of rumen microbiota}

After quality filtering, we obtained 578,207 microbial sequences from rumen samples, of which 4,066 were chimeric reads that were removed from further analysis. The mean sequence read length was 253 bp. We identified 1675 OTUs by OTU clustering of sequences with sequence similarity greater than $97 \%$. Of these 1597 OTUs were found, in both treatments, while 46 and 32 OTUs were endemic to the CS and WS treatments, respectively. We analyzed the richness and diversity of the microbial community using a dilution curve, the Shannon index, and the Chao index. The dilution curve tended to be flat, indicating that the sequencing data reached saturation and the amount of data and depth of sequencing were sufficient.

Hierarchical cluster analysis and principal coordinate analysis of samples for Beta diversity revealed significant differences in rumen microbial composition and structure between cattle receiving the two different diets, with the first two principal components accounting for $56.28 \%$ and $24.55 \%$ of the total variation, respectively (Fig. 3).

We analyzed the rumen microbial community compositions of beef cattle under the two treatments. Bacteroidetes accounted for $72.39 \%$ and $58.73 \%$ of the total bacteria in the WS and CS treatments, respectively. Firmicutes accounted for $19.20 \%$ and $24.24 \%$ of the total bacteria, respectively.

Proteobacteria accounted for $3.18 \%$ and $11.96 \%$ of the total bacteria, respectively. These were the dominant phyla in the two treatments and accounted for more than $90 \%$ of the total sequences (Fig. 4A). The abundance of Bacteroidetes in the WS treatment was significantly higher than that in the CS treatment $(P=0.005)$, the abundance of Proteobacteria in the WS treatment was significantly lower than that in the CS treatment $(P=0.045)$, but there was no significant difference in the abundance of Firmicutes between the two treatments (Fig. 4B). At the genus level, Prevotella_1 accounted for $49.22 \%$ and $35.84 \%$ of the total bacterial genera, Rikenellaceae_RC9_gut_group accounted for $6.70 \%$ and $6.23 \%$ of the total bacterial genera, Succinivibrionaceae_UCG-002 accounted for $2.37 \%$ and $10.52 \%$ of the total bacterial genera, Norank_f_F082 accounted for $3.54 \%$ and $4.92 \%$ of the total genera, and Prevotellaceae_UCG-001 accounted for $3.54 \%$ and $2.36 \%$ of the total genera, under the WS and CS treatments, respectively, and these were the dominant genera (Fig. 4C). The relative abundance of Prevotella_1 in the CS treatment was significantly lower than that in the WS treatment $(P=0.013)$, while the relative abundances of Succinivibrionaceae_UCG-002 and Succiniclasticum was significantly higher in the CS treatment than those in the WS treatment $(P=0.045$ and $P=0.045$, respectively).

Rikenellaceae_RC9_gut_group showed the same relative abundance between the two treatments $(P=$ 0.940) (Fig. 4D).

\subsection{Effects of different diets on the correlation of bacterial genera and VFA concentrations}


We analyzed the correlation between rumen flora and VFAs. As shown in Fig. 5, Prevotella_ 1 was positively correlated with acetic acid and ethylene/propylene ratio, Succinivibrionaceae_UCG-002 was positively correlated with propionic acid and butyric acid, and negatively correlated with $\mathrm{pH}$, Succiniclasticum was positively correlated with $\mathrm{pH}$ and acetate propionate ratio, and norank_f_F082 and Rikenellaceae_RC9_gut_group were positively correlated with $\mathrm{pH}$ and propionic acid and butyric acid.

\subsection{Effects of different diets on metabolic pathways in beef cattle}

We predicted the functions of rumen microbiota to analyze the difference between the two treatments. As shown in Fig.6A, there were significantly more OTUs associated with the functions of Amino Acid Metabolism, Nucleotide Metabolism, and Metabolism in the WS treatment than in the CS treatment $(P=$ $0.002, P=0.018, P=0.025$, respectively). The CS treatment produced significantly higher numbers of OTUs associated with Membrane Transport and Genetic Information Processing functions than the WS treatment $(P=0.007, P=0.004$, respectively).

We next analyzed the correlation between rumen microorganisms and metabolic pathways. As shown in Fig.6B, Succinivibrionaceae_UCG-002 was negatively correlated with Carbohydrate Metabolism, Glycan Biosynthesis and Metabolism, and Metabolism of Cofactors and Vitamins. Prevotellaceae_UCG-003 was positively correlated with Amino Acid Metabolism, Carbohydrate Metabolism, Energy Metabolism, Lipid Metabolism, Genetic Information Processing, Membrane Transport, and Metabolism of Cofactors and Vitamins. Ruminococcus_2 was positively correlated with Amino Acid Metabolism, Carbohydrate Metabolism, Energy Metabolism, and Lipid Metabolism.

\section{Discussion}

As the main source of nutrients for ruminants, roughage plays an important role in stimulating rumination and chewing, maintaining normal $\mathrm{pH}$ of rumen fluid and normal fermentation of rumen microbiota, and promoting digestion and metabolism of nutrients. Studies have shown that the utilization rate of animal feed can be improved by the regulating the rumen microbiota through dietary alterations [14]. Volatile fatty acids produced by fermentation of rumen carbohydrates are the main source of energy for ruminants, accounting for more than $75 \%$ of the total metabolic energy [8]. We therefore studied the effects of whole plant corn silage and corn straw silage on growth performance, rumen microbiota, and VFAs of beef cattle. The daily gain of beef cattle under the CS treatment was lower than that of cattle under the WS treatment. This may be due to the low nutritional value of corn straw and the high content of neutral detergent fibre (NDF) and Acid Detergent Fiber (ADF), as, too high cellulose content reduces the retention time of feed in the rumen and accelerates the movement of chyme in the intestine, thus reducing the digestibility of nutrients in beef cattle[15]. The digestibility of straw is only 20-30\% [16]. When beef cattle consume roughage comprising only corn straw silage, it is difficult for them to meet their maintenance needs, which affects growth performance. Khaing et al. [17] showed that replacing Napier grass with whole-plant corn silage increases goat weight gain, feeding and digestion. Zaralis et al. [4] showed that when whole-plant corn silage was added to the diet of fattening cattle, the feed intake of 
beef cattle could be increased, thus improving production performance. In our experiment, feeding wholeplant corn silage improved the production performance of beef cattle, similar to the results of the above study.

$\mathrm{pH}$ is an important indicator of rumen fermentation and can be used to determine whether rumen fermentation is normal or not. The normal pH of rumen fermentation is $\mathrm{pH}$ 6-7 [18]. Yang et al. [19] showed that a diet containing a high proportion of concentrate and lack of proper fiber caused accumulation of VFAs in the rumen and decreased the $\mathrm{pH}$ of rumen fluid. Rumen fluid $\mathrm{pH}$ lower than 5.8 is considered rumen acidosis [20]. In this experiment, cattle rumen $\mathrm{pH}$ during the two treatments was in the normal range and had no negative effect on rumen fermentation. The final product of rumen fermentation of dietary carbohydrates is VFAs, mainly acetic acid, propionic acid, and butyric acid. Lechartier et al. [21] found that increasing the dietary roughage level improved average rumen $\mathrm{pH}$, decreased the time and area of rumen $\mathrm{pH}<5.8$, and increased the rumen acetate to propionate $(\mathrm{A} / \mathrm{P})$ ratio. Ruminants fed diets with more crude fiber produced more rumen acetic acid, while diets rich in nonfiber carbohydrates contributed to the fermentation of propionic acid. The ratio of acetic acid to propionic acid affects energy utilization rate of energy and the location of energy storage. Acetic acid is the precursor of fat biosynthesis in ruminants while propionic acid is an important precursor of glucose biosynthesis; propionic acid fermentation can therefore provide more energy for the body and help livestock gain weight. In our experiment, acetic acid content and A/P ratio under the CS treatment were significantly higher than those under the WS treatment, but there was no significant difference in propionic acid or butyric acid content. This may be due to the higher fiber content of the CS treatment, which also caused lower daily weight gain in the CS group.

We evaluated the composition of Simmental hybrid bovine microbiota and its correlation with rumen metabolites. There was a significant difference in the Chao a-diversity index of rumen microbiota between the two treatment groups, and the community compositions of the two treatments were distinctly separated in $\beta$-diversity analysis, indicating significant differences in the structure and composition of microbial communities between the two treatments. At the phylum level, the dominant bacteria in the two treatment groups were Bacteroidetes and Firmicutes, which was similar to the results of Ley et al. [22]. These two bacterial phyla are found in the rumen of different ruminants, indicating that they play an important role in the rumen. The rumen of beef cattle fed with two different silages had a higher relative abundance of Bacteroidetes than of Firmicutes, similar to the results of Jami and Mizrahi [23] where the relative abundance of Bacteroidetes (50\%) was higher than that of Firmicutes (43\%) when the rumen $\mathrm{pH}$ was about 6.51 . Therefore, the rumen of ruminants fed on roughage is dominated by Bacteroides rather than Firmicutes in the range of normal $\mathrm{pH}$. Firmicutes produce a variety of lipases, proteases, cellulases, and other extracellular enzymes, thereby hydrolyzing complex macromolecular compounds, such as fats, proteins, amino acids, hemicellulose, cellulose, and sugars [24]. Bacteroidetes are mainly involved in the degradation of complex macromolecular organic compounds, such as carbohydrates to monosaccharides, which are then hydrolyzed into small acetic acid molecules, lactic acid, and succinic acid [25]. Bacteroidetes and Firmicutes can be used as important microbial indicator for evaluating the energy needs of ruminants [26]. In this experiment, feeding whole-plant corn silage 
significantly increased the relative abundance of Bacteroides and the degradation of macromolecules, which may improve the production performance of beef cattle. At the genus level, Prevotella is the most widespread and most abundant genus of bacteria in the rumen [27]. Prevotella function in hemicellulose degradation with high activity and can adjust bacterial numbers according to differences in dietary structure [28]. Co-culture of Prevotella and fiber-degrading bacteria can improve the utilization of plant hemicelluloses (pectin and xylan), thus promoting the degradation of fiber in the rumen. Prevotella also play an important role in the degradation of non-fibrous polysaccharides and proteins in plants [29]. The main metabolites of succinic acid bacteria are acetic acid and succinic acid, which can be fermented and converted into propionate [30]. In this study, feeding whole-plant corn silage significantly increased the relative abundance of Prevotella_1 and decreased the relative abundance of Succinivibrionaceae_UCG002, indicating that feeding whole-plant corn silage could increase the degradation of both fibrous and non-fibrous carbohydrates and that there was resource competition among rumen bacteria [31] because succinic acid bacteria can degrade non-structural carbohydrates in the bovine rumen [32]. The concentration of VFAs in the rumen is related to the rumen microbiota [29]. In this experiment, Prevotella_1, Succinivibrionaceae_UCG-002, and Rikenellaceae_RC9_gut_group were positively correlated with VFAs, indicating that these bacteria play an important role in the biosynthesis of VFAs and energy absorption and utilization, similar to the results of Peta [33] and Hanage [34]. We need to further explore this correlation, and studies have shown that a small number of species may have a strong effect on rumen fermentation parameters [35].

Microbiota function in host metabolism mainly through the use of carbohydrates that can not be utilized and absorbed by the host itself, including plant polysaccharides (such as resistant starch, cellulose, hemicellulose, colloid), oligosaccharides (such as oligofructose, inulin, etc.), and insoluble sugars, as well as endogenous mucus produced by epithelial cells, producing final products that participate in the metabolic processes of the host body [36]. The rumen microbiota is a symbiotic complex of microorganisms, which is not only an important source of protein in ruminants but also the main energy source for ruminants to produce VFAs through fiber fermentation. Therefore, rumen bacteria represent a huge pool of biological resources, and it is very important to actively explore the functional genes of rumen microbiota that are closely related to important nutritional and physiological functions, such as carbohydrate transport and metabolism, amino acid transport and metabolism, and production of VFAs. Through correlation analysis, we found that ruminant metabolic pathways were closely related to Succinivibrionaceae_UCG-002, Prevotellaceae_UCG-003, and Ruminococcus_2. Succinivibrionaceae_UCG002 belongs to the hemicellulose-degrading bacteria. Studies have shown that the rumen Succinivibrionaceae content of low-methane-producing cattle is four times higher than that of highmethane-producing cattle [37]. Carbohydrates are fermented to produce hydrogen and methane, and Succinivibrionaceae may help to reduce interspecific hydrogen transfer and methane production. In our experiment, Succinivibrionaceae were negatively correlated with carbohydrate metabolism, similar to the results of the above study. Prevotellaceae_UCG-003 can use hemicellulose and play an important role in protein metabolism and starch degradation [38,43]; they promote the heredity and metabolism of most microorganisms [39]. In our correlation analysis, Prevotellaceae_UCG-003 was positively correlated with 
Amino Acid Metabolism, Carbohydrate Metabolism, Gene Information Processing, and Biofilm Transport. Ruminococcus_2 belongs to the rumen coccidiaceae, which can hydrolyze and ferment carbohydrates and play an important role in rumen fermentation [40]. In our experiment, Ruminococcus_2 was positively correlated with Amino Acid Metabolism and Carbohydrate Metabolism. We analyzed the differences in metabolic pathways of rumen microbiota under the two diet treatments and found that after feeding whole-plant corn silage, the functions of Amino Acid Metabolism, Nucleotide Metabolism, and Metabolism were significantly up-regulated, while the functions of Biofilm Transport and Gene Information Processing were significantly down-regulated compared with those in cattle fed corn straw silage. This may be due to the higher relative abundance of Prevotella and Succinivibrionaceae UCG-002 in the WS group than in the CS group. Prevotella can eventually degrade protein into various peptides, which can be effectively absorbed by the rumen and promote the formation of bacterial protein. Prevotella therefore play an important role in rumen protein metabolism [41]. Succinivibrionaceae_UCG 002 was negatively correlated with Amino Acid Metabolism, Nucleotide Metabolism, and Metabolism and may cause the up-regulation of these processes. Rumen microbiotal composition is very complex and there are a large number of anaerobic microorganisms that cannot be cultured, meaning we are unable to study their specific functions. The nutrition level of the body, health status, environmental factors, and other complex factors affect rumen microbiota, so the specific regulatory mechanisms of microbiotal involvement in amino acid metabolism and carbohydrates metabolism and other metabolic pathways remains to be explored.

\section{Materials And Methods}

\subsection{Animals, diet, and experimental design}

All experimental procedures in this study were approved by the Institutional Animal Ethics Committee of Henan Agricultural University (approval number: HENAU-2016-015). The experiment was conducted at Henan Hengdu Xianan Cattle Development Limited Company. The whole-plant corn silage and corn straw silage were produced on the experimental cattle farm, and were stored until the start of the experiment. The storage time is 75 days. Select 60 healthy Simmental hybrid cattle $(448.5 \pm 18.37 \mathrm{~kg})$ and randomly divide them into two groups ( 6 replicates in each group and 5 cattle in each replicate). Two diets were designed in the experiment. One diet was supplemented with $27.05 \%$ whole-plant corn silage, and the other diet was supplemented with $27.05 \%$ corn straw silage. The detailed ingredient composition and nutrient content of the investigated diets are presented in Table 1. The pre-trial period was 10 days, and the formal trial period was 80 days. All cattle were under unified management; feed and drinking water were supplied, with feeding at 8:30 and 14:30 every day, barn spray disinfection and manure removal were carried out regularly, and feeding management and immunization were performed according to routine methods. Average daily feed intake (ADFI) is calculated by recording the amount of leftover feed. Animals of each group were weighed before the morning feeding on the first and 90th days of the trial, recorded as the initial weight and the last weight, to calculate average daily gain (ADG). Feed to gain (F: $\mathrm{G})$ ratio was recorded according to the average daily dry matter intake and ADG. 


\subsection{Sample collection}

At the end of the experiment, one cattle in each replicate was selected for sampling (6 samples in each group). Ruminal fluid was collected $3 \mathrm{~h}$ after the morning feeding on the last day of this experiment using an oral stomach tube according to Shen et al.[42]. $50 \mathrm{~mL}$ rumen fluid was collected from each cattle, and the rumen fluid was filtered with four layers of gauze. The fluid $\mathrm{pH}$ was measured immediately. The samples were immediately flash-frozen in liquid nitrogen and stored at $-80 \circ \mathrm{C}$ for DNA extraction and measuring the volatile fatty acids (VFAs) according to Hu et al. [43] and to determine the ammonia nitrogen according to Broderick and Kang [44].

\subsection{Determination of rumen fermentation parameters.}

Rumen fluid pH was measured using a portable pH meter (Sartorius PB-10, Sartorius, Göttingen, Germany) during fluid collection. Cryopreserved rumen fluid samples were thawed at $4^{\circ} \mathrm{C}$ and mixed thoroughly by vortexing. Aliquots of rumen fluid $(5 \mathrm{~mL})$ were centrifuged at $3,000 \times \mathrm{g}$ for $10 \mathrm{~min}, 1 \mathrm{~mL}$ of supernatant was placed in a $1.5 \mathrm{~mL}$ centrifuge tube, and $0.2 \mathrm{~mL}$ of a metaphosphoric acid solution containing the internal standard 2-ethyl butyric acid was added. Samples were mixed, placed in an icewater bath for $30 \mathrm{~min}$, and then centrifuged at $10,000 \times \mathrm{g}$ at $4^{\circ} \mathrm{C}$. The supernatant was placed in a new $1.5 \mathrm{~mL}$ centrifuge tube and stored in a $4^{\circ} \mathrm{C}$ refrigerator for testing. VFA concentration was determined by gas chromatography (Varian 450, Agilent Technologies China Co., Ltd., China).

\subsection{DNA extraction and 16S rRNA gene sequencing}

DNA was extracted from fluid using a commercially available E.Z.N.A DNA Stool Mini kit (Omega,USA). DNA concentration was determined using a Nano Drop 2000(Thermo Fisher Scientific, Waltham, MA, USA), and DNA integrity was checked using $1 \%$ agarose gel electrophoresis. The V3-V4 hypervariable region of the bacterial 16S rRNA gene was amplified by PCR using primers 338F ( 5 '-

ACTCCTACGGGAGGCAGCAG-3') and 806R (5'-GGACTACHVGGGTWTCTAAT-3') and the following program: 3 min denaturation at $95^{\circ} \mathrm{C} ; 27$ cycles of 30 s denaturation at $95^{\circ} \mathrm{C}, 30 \mathrm{~s}$ annealing at $55^{\circ} \mathrm{C}$, and 45 s elongation at $72{ }^{\circ} \mathrm{C}$; and a final extension at $72{ }^{\circ} \mathrm{C}$ for $10 \mathrm{~min}$. PCR reactions were performed in triplicate with each $20 \mathrm{~mL}$ reaction mixture containing $4 \mathrm{~mL} 5 \times$ FastPfu Buffer, $2 \mu \mathrm{L} 2.5 \mathrm{mM}$ dNTPs, 0.8 $\mu \mathrm{L}$ of each primer $(5 \mu \mathrm{M}), 0.4 \mu \mathrm{L}$ FastPfu Polymerase, and $10 \mathrm{ng}$ template DNA. The resulting PCR products were extracted from a $2 \%$ agarose gel and further purified using an AxyPrep DNA Gel Extraction Kit (Axygen Biosciences, Union City, CA, USA). A paired-end amplification library was constructed and sequenced using the Illumina Miseq platform by Shanghai Meiji Biopharmaceutical Technology Co., Ltd., China. The raw reads were deposited into the NCBI Sequence Read Archive (SRA) database under accession number SRP 298526.

\subsection{Bioinformatics analysis of sequencing data}

Raw fastq files were quality-filtered by Trimmomatic and merged by FLASH: the reads were truncated at any site receiving an average quality score $<20$ over a 50 bp sliding window; sequences whose overlap 
was longer than $10 \mathrm{bp}$ were merged according to their overlap with no more than $2 \mathrm{bp}$ mismatch; sequences of each sample were separated according to barcodes (exact matches) and primers (allowing for two nucleotide mismatches). Reads containing ambiguous bases were removed. Operational taxonomic units (OTUs) were clustered with 97\% similarity cutoff using UPARSE (version 7.1; http://drive5.com/uparse/) with a novel 'greedy' algorithm that performs chimera filtering and OTU clustering simultaneously. Sample biodiversity was calculated using the ACE, Chao1, and Shannon indices. $\mathrm{R}$ language tools were used to generate graphs and colony histograms. Changes in relative abundance of bacteria are shown using column charts [45]. Weighted Unifrac principal coordinate analysis [46] and hierarchical cluster analysis of Bray-Curtis samples based on OTU level were used to summarize the composition of rumen microbiota [47]. To determine the effect of posterior segment microbiota interacting with Apparent performance, redundancy analysis (RDA) was performed at the genus level using the $\mathrm{R}$ language vegan packet [48].Three levels of metabolic pathway information were obtained using PICRUSt [49] for Pathway, and abundance tables for each level were obtained and analyzed by majorbio Co., Ltd., China.

\subsection{Statistics and analysis}

Analyses of data were performed using the software SPSS18.0 (IBM, New York, NY, USA). Differences between means were assessed using independent sample t-test $P<0.05$ considered statistically significant.

Table 1 Composition and nutrient levels of diet of beef cattle[DM basis \% 


\begin{tabular}{|c|c|c|}
\hline Items & $W^{a}$ & $C S^{a}$ \\
\hline Whole corn silage & 27.05 & \\
\hline Corn stalk silage & & 27.05 \\
\hline Peanut seedlings & 12.65 & 12.65 \\
\hline Distiller's grains & 10.18 & 10.18 \\
\hline Bean dregs & 2.13 & 2.13 \\
\hline Molasses & 4.36 & 4.36 \\
\hline Concentrate supplement ${ }^{\mathrm{b}}$ & 43.63 & 43.63 \\
\hline Total & 100.00 & 100.00 \\
\hline NEmf ${ }^{c}(\mathrm{MJ} / \mathrm{kg})$ & 6.51 & 5.98 \\
\hline $\mathrm{CP}^{\mathrm{C}}$ & 12.63 & 12.25 \\
\hline $\mathrm{ADF}^{\mathrm{C}}$ & 24.05 & 28.28 \\
\hline $\mathrm{NDF}^{\mathrm{C}}$ & 34.50 & 42.04 \\
\hline $\mathrm{Ca}^{\mathrm{C}}$ & 0.31 & 0.31 \\
\hline $\mathrm{P}^{\mathrm{c}}$ & 0.14 & 0.16 \\
\hline
\end{tabular}

a whole-plant Corn silage group (WS), Corn Straw group (CS).

$\mathrm{b}$ Ingredients of concentrate supplement were $63 \%$ corn, $15 \%$ DDGS, $12 \%$ sesame meal,2\%cottonseed meal, $3 \% \mathrm{NaHCO}, 1 \% \mathrm{MgO}, 1 \% \mathrm{NaCl}$ and $3 \%$ premix. Contained the following per $\mathrm{kg}$ of the premix: $\mathrm{Fe}$ 1000mg, Zn 450mg, Cu 105mg, Se 2mg, I 50mg, Co 1mg, VA 3000, VD 1000IU, VE10mg

${ }^{\mathrm{C}}$ NEmf: Combined net energy, CP: Crude protein, CF: Crude fiber, ADF: Acid Detergent Fiber, NDF: Neutral detergent fibre, Ca: Calcium, P: Phosphorus.

\section{Conclusion}

(1) Feeding whole-plant corn silage can increase the daily gain and decrease the feed weight ratio of beef cattle. (2) Feeding whole-plant corn silage induces changes in rumen microbiota and improves rumen fermentation in beef cattle. (3) Feeding whole-plant corn silage can improve amino acid metabolism, nucleotide metabolism, and gene functions associated with metabolism, but specific mechanisms need to be further studied. (4) We speculate that the improvement in growth performance of beef cattle fed whole-plant corn silage may be related to changes in rumen microbiota that improve rumen metabolism 
by increasing the metabolism of amino acids and nucleotides; however, specific mechanisms need to be further studied.

\section{Declarations}

\section{Ethics approval and consent to participate}

Not applicable.

\section{Consent for publication}

All authors consent for publication.

\section{Availability of data and material}

The authors declare that data supporting the findings of this study are available within the article.

\section{Competing interests}

The authors declare that they have no competing interests.

\section{Funding and acknowledgement}

Thanks for financial support of China Agriculture Research System of MOF and MARA and Demonstration project for development and utilization of high-quality green roughage resources (16200157) and Science and Technology Innovation Project of Henan Agricultural University (No.KJCX2019A07).

\section{Authors' contribution}

Y.C. and H.L. performed experiments and analyzed data. Z.G., J.X. and B.L. participated in the data collection. M.G., X.Y., J.N., and X.Z. assisted with animal experimentation. S.M. and D.L. provided advice in design and performance of experiments. Y.C. and H.L. wrote the manuscript draft. Y.S. and Y.H.S.supervised the study. All of us read and approved the final manuscript.

\section{References}

1. Moloney AP, Drennan MJ. Characteristics of fat and muscle from beef heifers offered a grass silage or concentrate-based finishing ration. Livestock Science. 2013;152(2-3):147-153.

2. O'Mara FP, Fitzgerald JJ, Murphy JJ, Rath M. The effect on milk production of replacing grass silage with maize silage in the diet of dairy cows. Livestock Production Science. 1998.

3. Hameleers A. The effects of the inclusion of either maize silage, fermented whole crop wheat or ure-treated whole crop wheat in a diet based on a high-quality grass silage on the performance of dairy 
cows. Grass \& Forage Ence. 2010;53(2):157-163.

4. Zaralis K, Nørgaard P, Helander C, Murphy M, Weisbjerg MR, Nadeau E. Effects of maize maturity at harvest and dietary proportion of maize silage on intake and performance of growing/finishing bulls. Livestock Science. 2014;168:89-93.

5. Hameleers A. The effects of the inclusion of either maize silage, fermented whole crop wheat or ure-treated whole crop wheat in a diet based on a high囚uality grass silage on the performance of dairy cows. Grass \& Forage Science. 1998;53(2):157-163.

6. Anantasook N, Wanapat M, Cherdthong A, Gunun P. Changes of microbial population in the rumen of dairy steers as influenced by plant containing tannins and saponins and roughage to concentrate ratio. Asian-Australasian journal of animal sciences. 2013;26(11):1583-1591.

7. $\quad \mathrm{Y} L, P P, T L, A$ W. The genome of archaeal prophage PsiM100 encodes the lytic enzyme responsible for autolysis of Methanothermobacter wolfeii. Journal of bacteriology. 2001;183(19):57885792.

8. $\quad \mathrm{NBE}$. Energy contributions of volatile fatty acids from the gastrointestinal tract in various species. Physiological reviews. 1990;70(2):319-425.

9. $\mathrm{S} K \mathrm{KE}, \mathrm{J} \mathrm{dVM}$. Prediction of ruminal $\mathrm{pH}$ from pasture-based diets. Journal of dairy science. 2002;85(5):1255-1266.

10. Russell JB, Rychlik JL. Factors that alter rumen ecology. Science. Science. 2001;292(5519):11191122.

11. Jami E, White BA, Mizrahi I. Potential Role of the Bovine Rumen Microbiome in Modulating Milk Composition and Feed Efficiency. PLOS ONE. 2014;9(1):e85423.

12. A HS, J CC, B OL, et al. Addressing Global Ruminant Agricultural Challenges Through Understanding the Rumen Microbiome: Past, Present, and Future. Frontiers in microbiology. 2018;9: 2161.

13. Liu C, Wu H, Liu S, Chai S, Meng Q, Zhou Z. Dynamic Alterations in Yak Rumen Bacteria Community and Metabolome Characteristics in Response to Feed Type. Frontiers in microbiology. 2019;10:1116.

14. Chao $Y$, Jianbin $L$, Xiaoyun $W$, et al. The response of gene expression associated with lipid metabolism, fat deposition and fatty acid profile in the longissimus dorsi muscle of Gannan yaks to different energy levels of diets. PloS one. 2017;12(11):e0187604.

15. lannaccone M, lanni A, Contaldi F, et al. Whole blood transcriptome analysis in ewes fed with hemp seed supplemented diet. Scientific Reports. 2019;9(1):16192. 


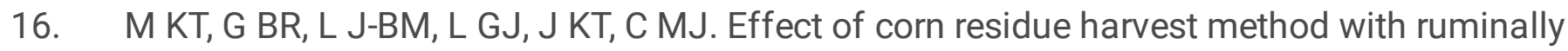
undegradable protein supplementation on performance of growing calves and fiber digestibility. Journal of animal science. 2017;95(12):5290-5300.

17. Khaing KT, Loh TC, Ghizan S, Halim RA, Samsudin AA. Feed intake, growth performance and digestibility in goats fed whole corn plant silage and Napier grass. 2015.

18. E NJ. Bovine acidosis: implications on laminitis. Journal of dairy science. 1997;80(5):1005-1028.

19. Z YW, A BK. Effects of physically effective fiber on chewing activity and ruminal pH of dairy cows fed diets based on barley silage. Journal of dairy science. 2006;89(1): 217-228.

20. $B$ PG, A BK, T M. Severity of ruminal acidosis in primiparous holstein cows during the periparturient period. Journal of dairy science. 2007;90(1):365-375.

21. $\quad C L, J-L P$. The effects of forage proportion and rapidly degradable dry matter from concentrate on ruminal digestion in dairy cows fed corn silage-based diets with fixed neutral detergent fiber and starch contents. Journal of dairy science. 2010;93(2):666-681.

22. Ley RE, Hamady M, Lozupone C, et al. Evolution of Mammals and Their Gut Microbes. 2008;320(5883):1647-1651.

23. Jami E, Mizrahi I. Composition and Similarity of Bovine Rumen Microbiota across Individual Animals. PLOS ONE. 2012;7(3):e33306.

24. St-Pierre B, Wright A-DG. Comparative metagenomic analysis of bacterial populations in three fullscale mesophilic anaerobic manure digesters. Applied Microbiology and Biotechnology. 2014;98(6):27092717.

25. Zhengbo Y, Rui C, Fan Y, et al. Effects of dairy manure and corn stover co-digestion on anaerobic microbes and corresponding digestion performance. Bioresource technology. 2013;128:65-71.

26. Xue $\mathrm{D}, \mathrm{Chen} \mathrm{H}$, Zhao $\mathrm{X}$, et al. Rumen prokaryotic communities of ruminants under different feeding paradigms on the Qinghai-Tibetan Plateau. Systematic and Applied Microbiology. 2017:227.

27. Sharma, Prasad, Singh, Bishisth. Effect of polyherbal preparation supplementation on immunity and udder health of periparturient Karan-Fries crossbred dairy cows. Journal of Applied Animal Research. 2014;42(2).

28. Zhi-Peng LI, Na J, Han-Lu L, et al. Analysis of Bacterial Diversity in Rumen of Sika Deer(Cervus nippon) fed Different Forages Using DGGE and T-RLFP. entia Agricultura Sinica. 2014;47(4):759-768.

29. Griswold KE, White BA, Mackie RI. Diversity of Extracellular Proteolytic Activities Among Prevotella Species from the Rumen. Current Microbiology. 1999;39(4):187-194. 
30. GYLSWYK NOv. Succiniclasticum ruminis gen. nov., sp. nov., a Ruminal Bacterium Converting Succinate to Propionate as the Sole Energy-Yielding Mechanism. International Journal of Systematic Bacteriology. 1995;45(2):297-300.

31. R MP, L STP, E WJ, A KL, C FH. Rumen microbiome from steers differing in feed efficiency. PloS one. 2015;10(6):e0129174.

32. Gemma H, Faith C, Siva G, Arjan J, Wayne Y, H JP. Erratum: Rumen microbial community composition varies with diet and host, but a core microbiome is found across a wide geographical range. Scientific reports. 2016;6:19175.

33. Petia K-D, Anne N, Rozita A, et al. Dietary Fiber-Induced Improvement in Glucose Metabolism Is Associated with Increased Abundance of Prevotella. Cell metabolism. 2015;22(6):971-982.

34. Holman DB, Gzyl KE. A meta-analysis of the bovine gastrointestinal tract microbiota. FEMS Microbiology Ecology. 2019;95(6):fiz072.

35. Hanage, P. W. Microbiology: Microbiome science needs a healthy dose of scepticism. Nature. 2014:247-248.

36. Malagelada GJR. Gut flora in health and disease. Lancet. 2003:512-519.

37. Wallace RJ, Rooke JA, McKain N, et al. The rumen microbial metagenome associated with high methane production in cattle. BMC Genomics. 2015;16(1):839.

38. Andreja R, Matjaž $\mathrm{P}$, Kiyoshi $\mathrm{T}$, et al. Unravelling the genetic diversity of ruminal bacteria belonging to the CFB phylum. Fems Microbiology Ecology. 2000(1):69-79.

39. Purushe J, Fouts DE, Morrison M, et al. Comparative Genome Analysis of Prevotella ruminicola and Prevotella bryantii : Insights into Their Environmental Niche. Microbial Ecology. 2010;60(4):721-729.

40. Rey M, Enjalbert F, Combes S, Cauquil L, Bouchez O, Monteils V. Establishment of ruminal bacterial community in dairy calves from birth to weaning is sequential. Journal of Applied Microbiology. 2014;116(2):245-257.

41. I.N.R.A, France. Conference: Altering Ruminai Nitrogen Metabolism to Improve Protein utilization. Nutrition Research. 1996;126(4 Suppl):1324S-1325S.

42. Shen J, Chai Z, Song L, Liu J, Wu Y. Insertion depth of oral stomach tubes may affect the fermentation parameters of ruminal fluid collected in dairy cows. Journal of dairy science. 2012;95(10):5978-5984.

43. Hu WL, Liu JX, Ye JA, Wu YM, Guo YQ. Effect of tea saponin on rumen fermentation in vitro. Animal Feed Science and Technology. 2005;120(3-4):333-339. 
44. Broderick G, Kang J. Automated simultaneous determination of ammonia and total amino acids in ruminal fluid and in vitro media. Journal of dairy science. 1980;63(1):64-75.

45. M SK, K JT, Bhaskar R, et al. Microbial profiles of liquid and solid fraction associated biomaterial in buffalo rumen fed green and dry roughage diets by tagged 16S rRNA gene pyrosequencing. Molecular biology reports. 2015;42(1):95-103.

46. Catherine L, E LM, Dan K, Jesse S, Rob K. UniFrac: an effective distance metric for microbial community comparison. The ISME journal. 2011;5(2):169-172.

47. Xiao-Tao J, Xin P, Guan-Hua D, et al. Illumina sequencing of $16 \mathrm{~S}$ rRNA tag revealed spatial variations of bacterial communities in a mangrove wetland. Microbial ecology. 2013;66(1):96-104.

48. Mu C, Yang Y, Su Y, Zoetendal E, Zhu W. Differences in Microbiota Membership along the Gastrointestinal Tract of Piglets and Their Differential Alterations Following an Early-Life Antibiotic Intervention. Frontiers in microbiology. 2017;8:797.

49. Langille MGI, Zaneveld J, Caporaso JG, et al. Predictive functional profiling of microbial communities using $16 \mathrm{~S}$ rRNA marker gene sequences. Nature Biotechnology: The Science and Business of Biotechnology. 2013;31(suppl. 1):814-821.

\section{Figures}

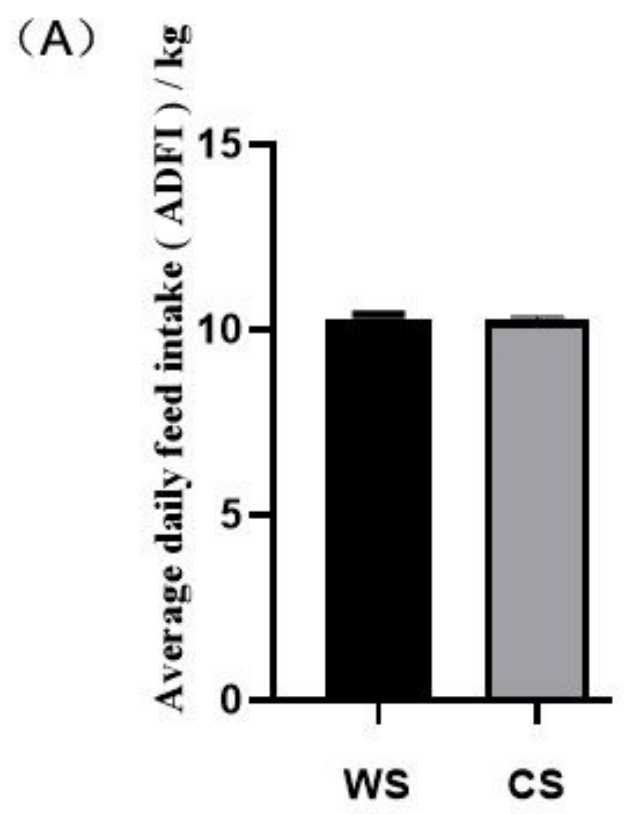

(B)

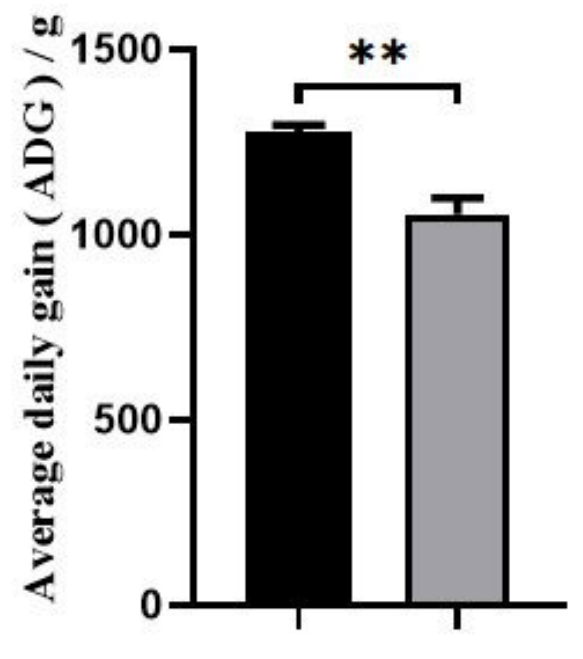

WS CS
(C)

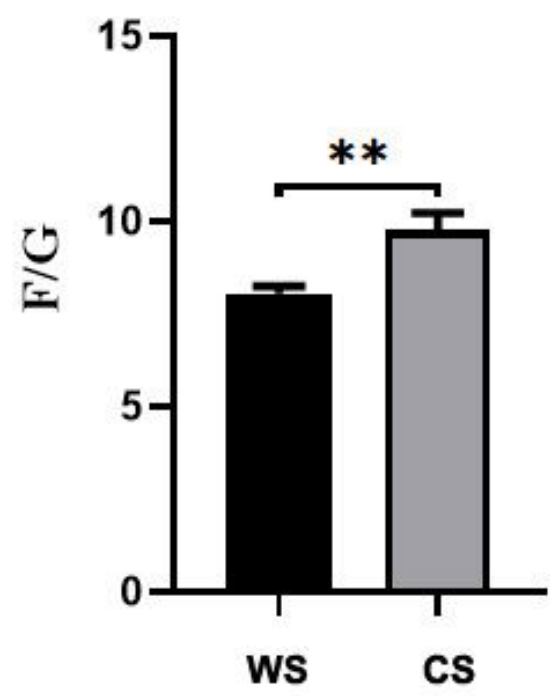

\section{Figure 1}

Effects of different treatments on growth performance of beef cattle. (A) Average daily feed intake, (B) Average daily gain, (C) Feed/Gain. $* \star 0.001<\mathrm{P}<=0.01$. 
(A)

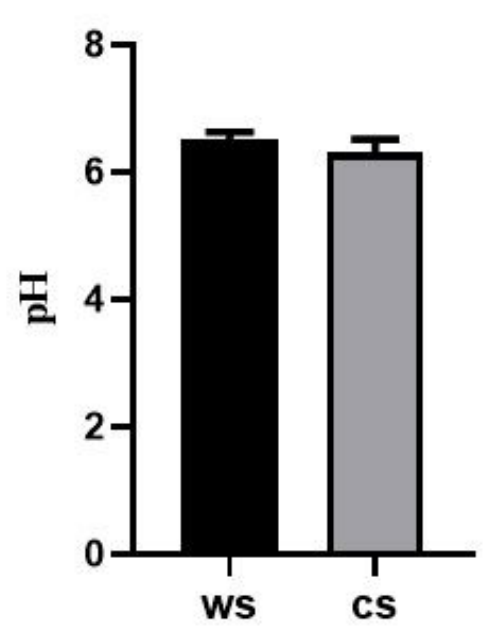

(D)

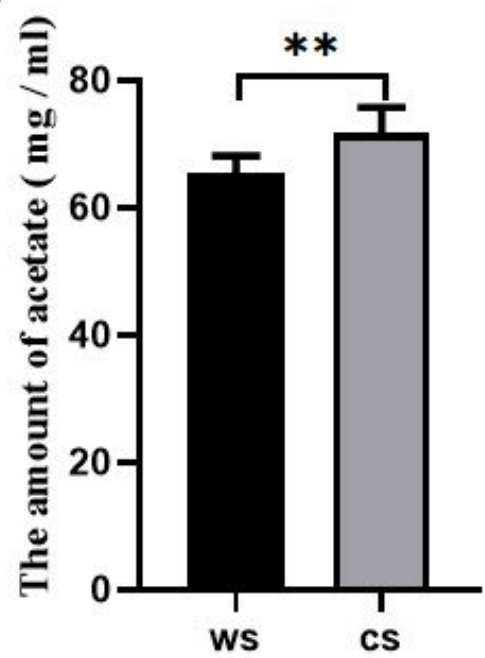

(B)

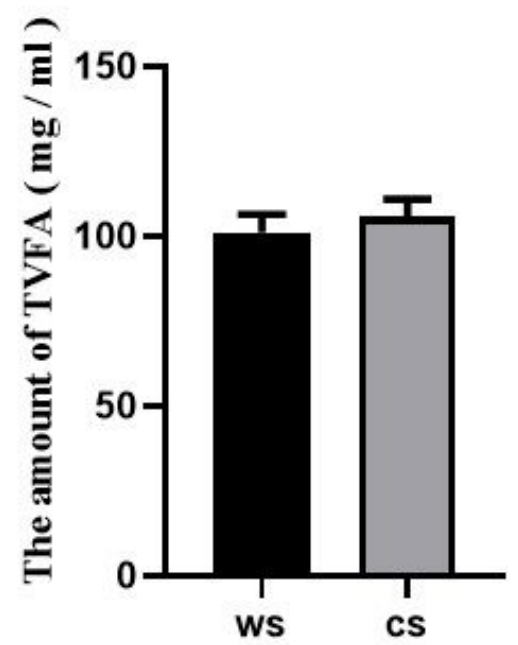

$(\mathrm{E})$

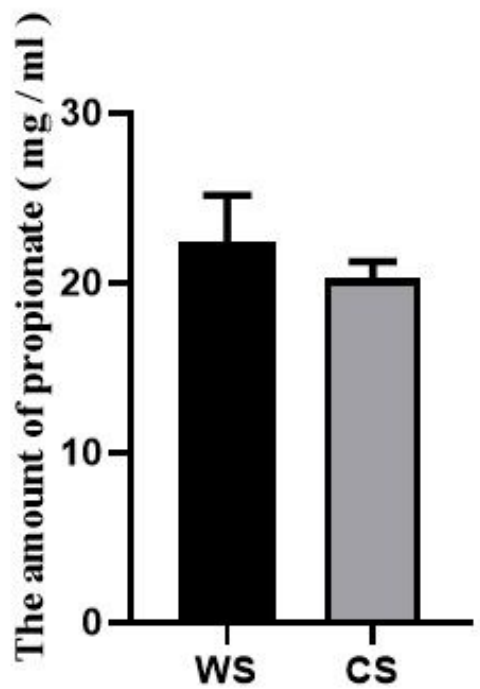

(C)

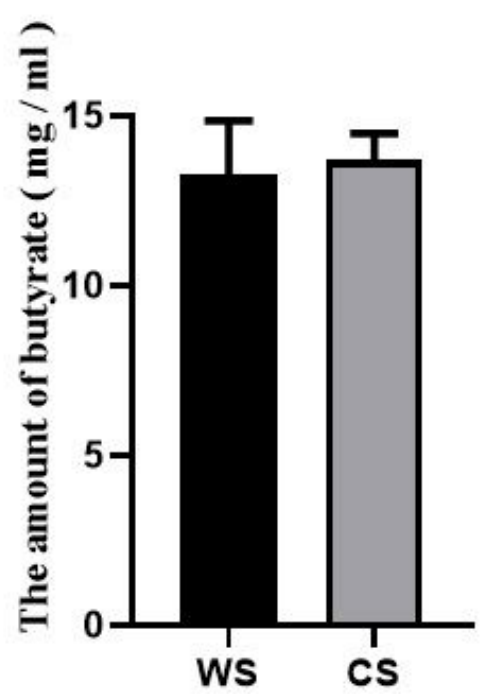

(F)

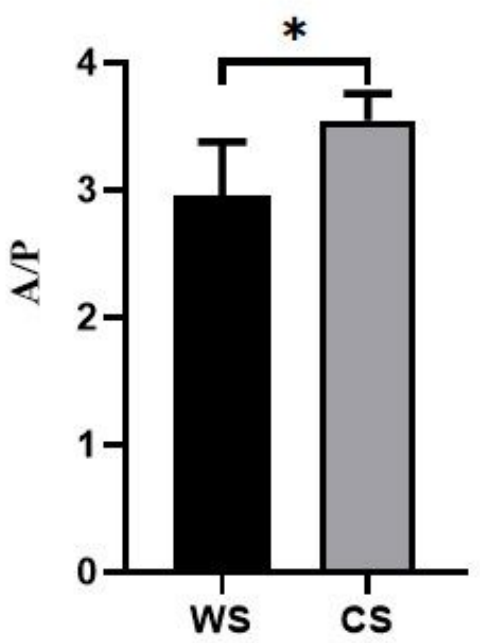

Figure 2

Effects of different diets on rumen volatile fatty acids of beef cattle. (A) $\mathrm{pH}$, (B) TVFA, (C) Butyrate, (D) Acetate, (E) Propionate, (F) A/P. * $0.01<P<=0.05$, ** $0.001<P<=0.01$. 
(A)

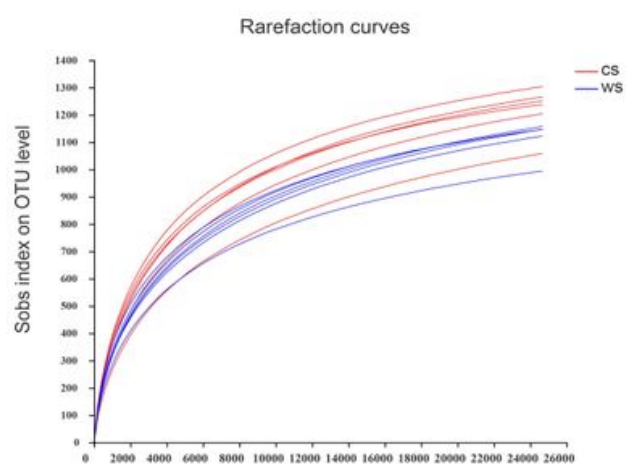

(B)

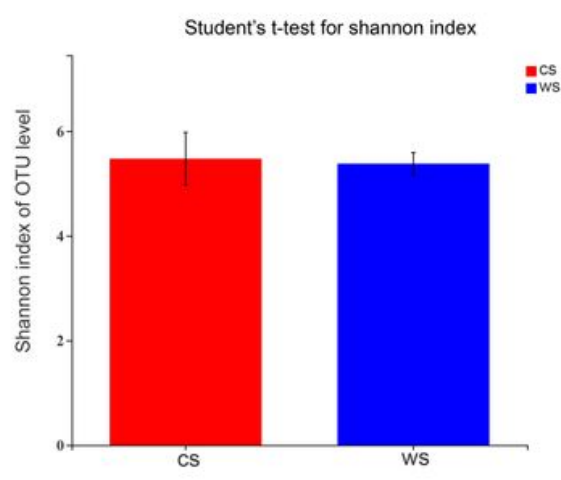

(C)

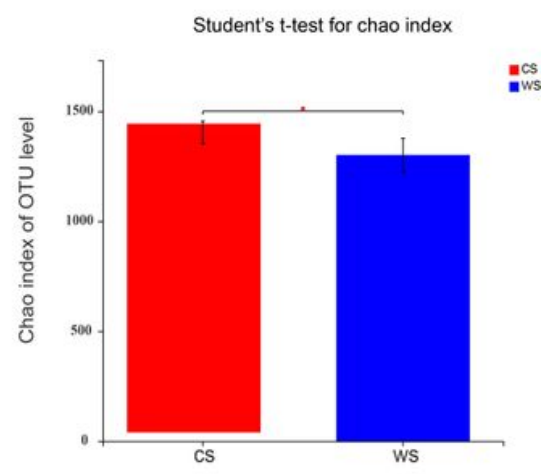

(D)

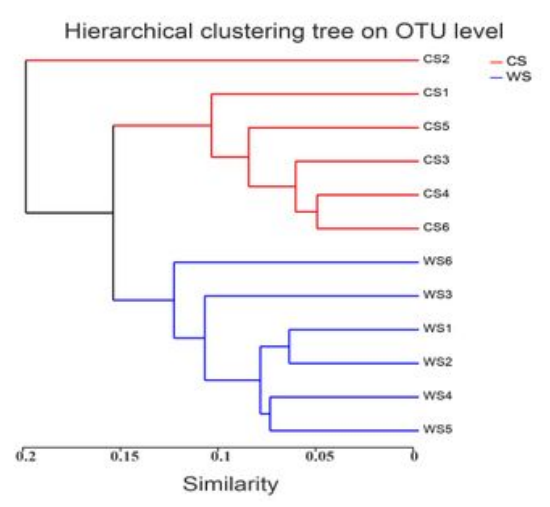

(E)

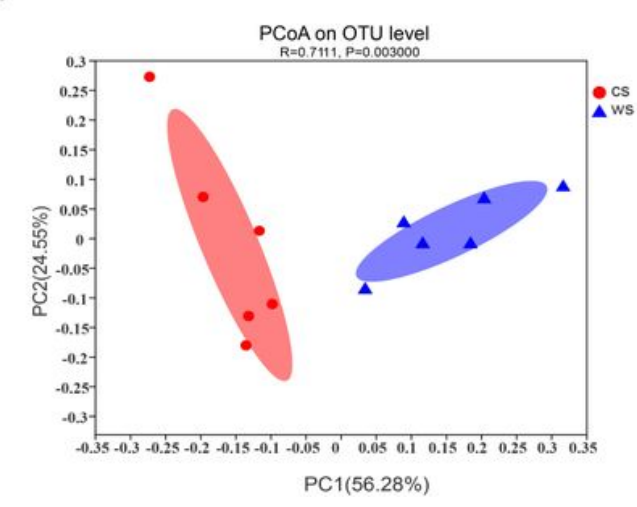

\section{Figure 3}

Effects of Different Diets on Rumen Microbial Alpha and Beta Diversity in Beef Cattle. (A) Rarefaction curve, (B) Shannon index, (C) Chao index, (D) Hierarchical clustering, (E) Principal Co-ordinates Analysis. Abbreviations: Whole-plant Corn silage group (WS), blue; Corn Straw group (CS), red. * $0.01<P<=0.05$, ** $0.001<\mathrm{P}<=0.01, * \star * \mathrm{P}<=0.001$. 
(A)

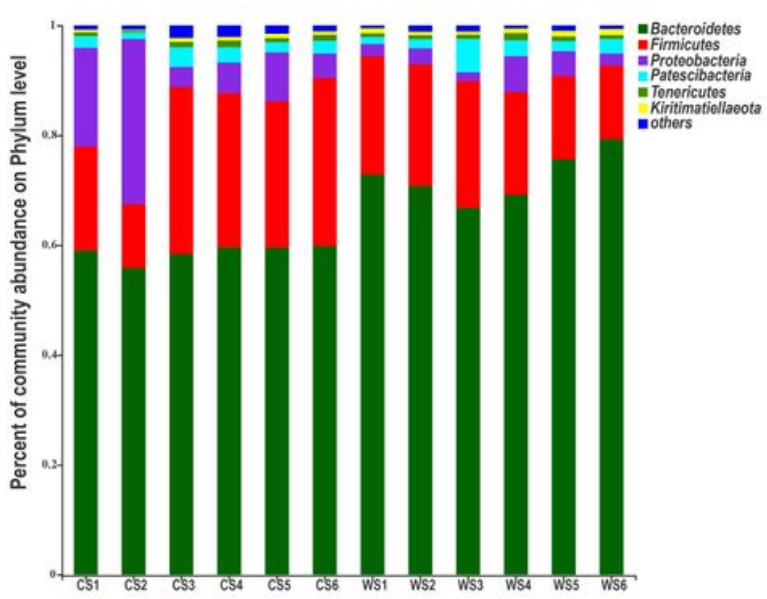

(C)

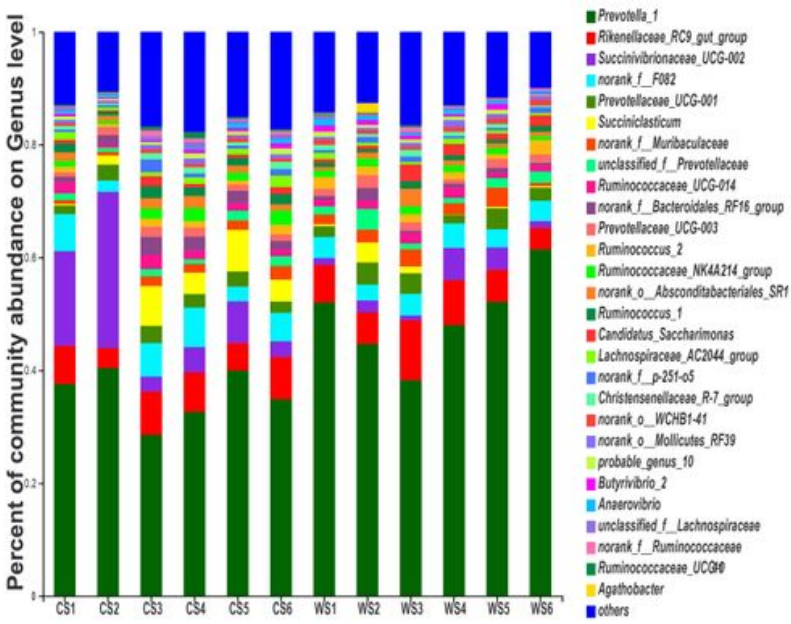

(B)

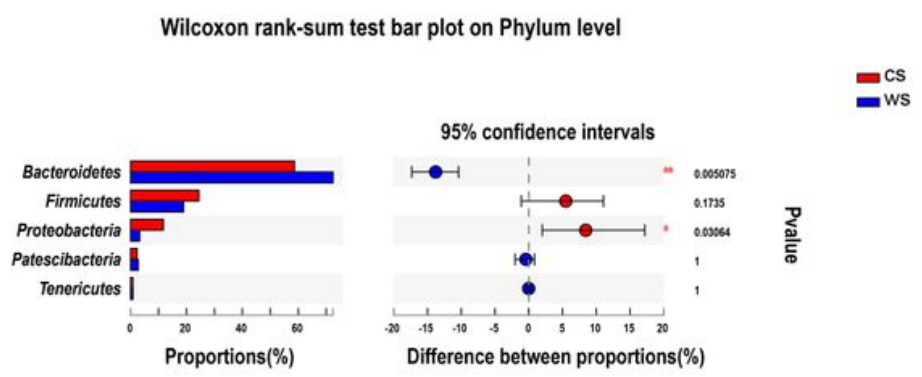

(D)

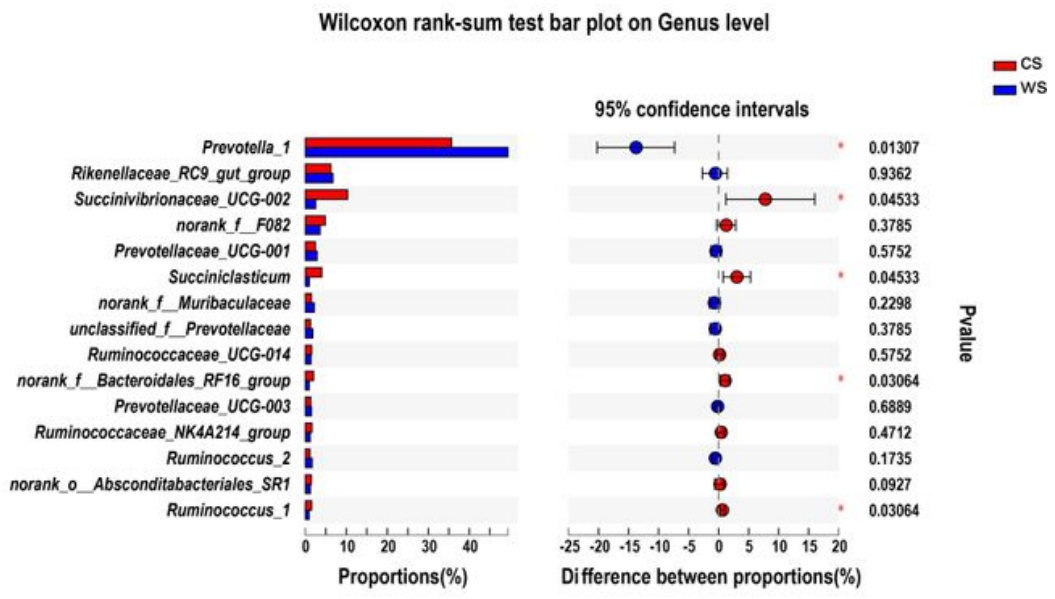

Figure 4

Analysis on the Composition and Difference of the Rumen Microbiota of Beef Cattle in Different Diets. (A) Microbiota Community at the Phylum Level, (B) The Composition Analysis of Microbiota Community at the Phylum Level, (C) Microbiota Community at the Genus Level, (D). The Composition Analysis of Microbiota Community at the Genus Level. Abbreviations: whole-plant Corn silage group (WS), blue; Corn Straw group (CS), red. * $0.01<\mathrm{P}<=0.05$, ** $0.001<\mathrm{P}<=0.01, * \star * \mathrm{P}<=0.001$. 
(B)
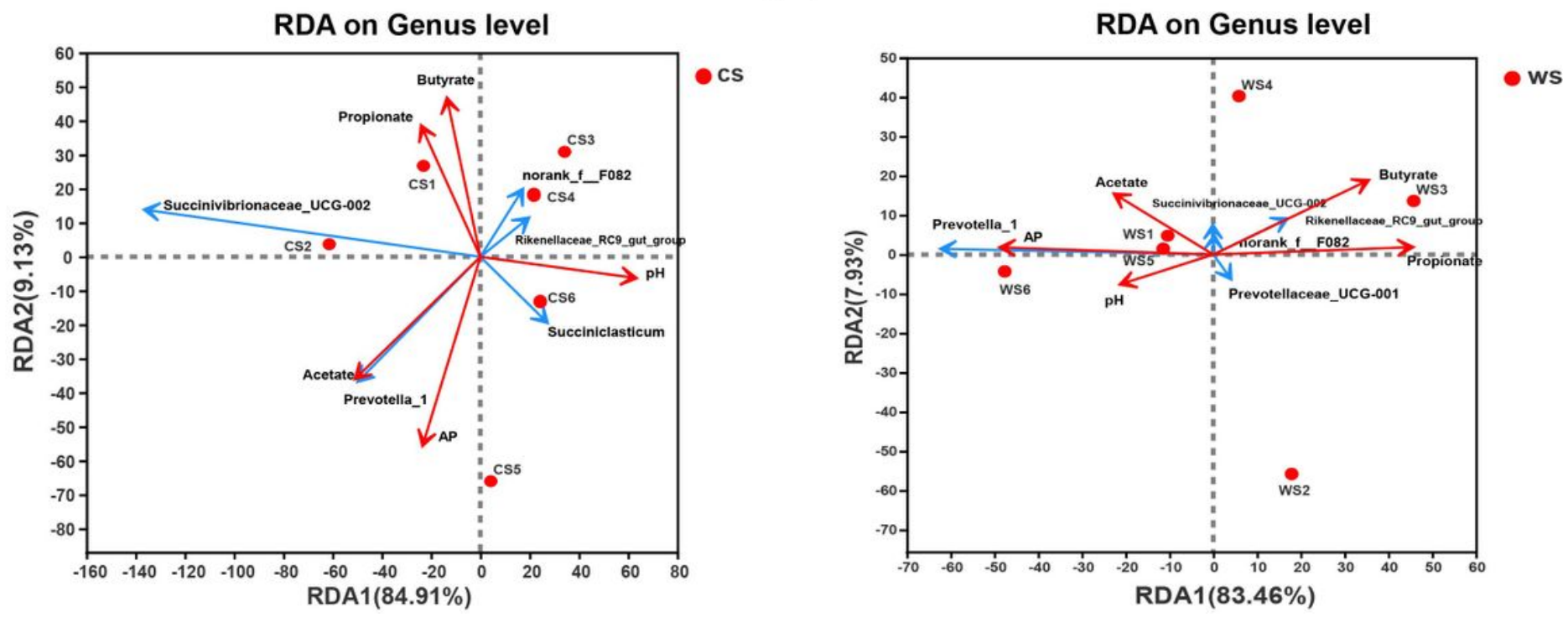

Figure 5

Association and model predictive analysis. (A) RDA analysis of microbial genus level in CS group, (B) RDA analysis of microbial genus level in WS group. Abbreviations: whole-plant Corn silage group (WS); Corn Straw group (CS).

(A)
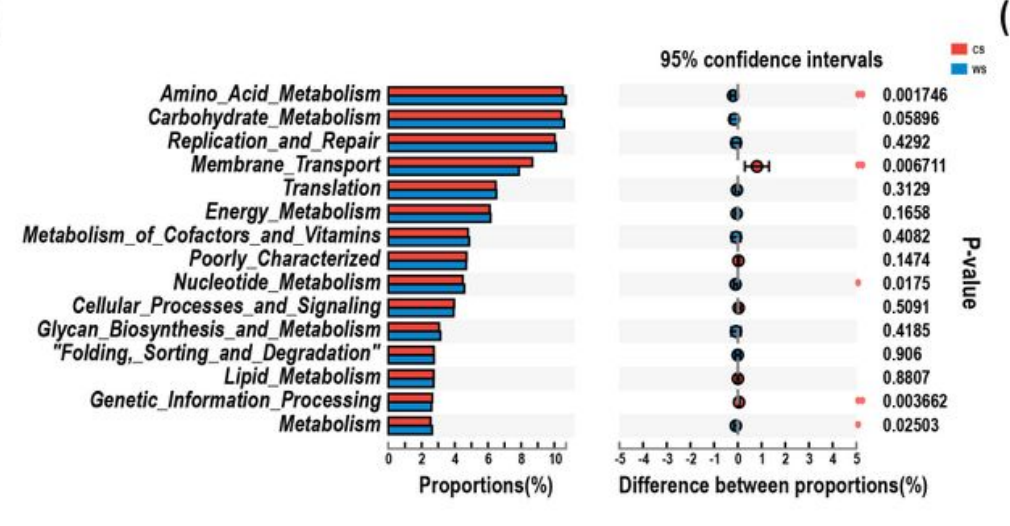

(B)

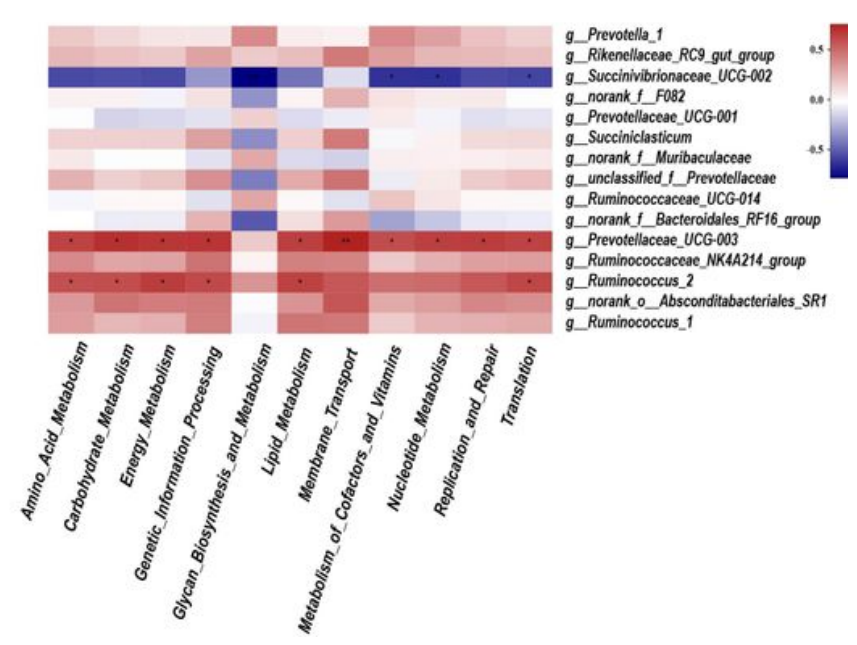

Figure 6

Effects of diets with different treatments on metabolic pathways of beef cattle. (A) The difference analysis of the top 15 metabolic pathways in the prediction of bacterial function between the two groups, (B) Heatmap of the correlation analysis was conducted between the top 15 bacterial genera and the metabolic pathways. Abbreviations: whole-plant Corn silage group (WS), blue; Corn Straw group (CS), red. $\star 0.01<\mathrm{P}<=0.05, * \star 0.001<\mathrm{P}<=0.01, * * * \mathrm{P}<=0.001$. 